\title{
The Association of Respiratory Morbidity and Assisted Ventilation Rates in Late Preterm Births after Premature Rupture of Membranes: A Retrospective Cohort Study in a Tertiary Center
}

\author{
Ji Su Seong, MD', \\ Soo Hyun Oh, MD', \\ Se Jin Lee, $M D^{1,2}$, \\ Jee Yoon Park, MD', \\ Kyung Joon $\mathrm{Oh}, \mathrm{MD}, \mathrm{PhD}^{1}$, \\ Joon-Seok Hong, MD, PhD \\ 'Department of Obstetrics and \\ Gynecology, Seoul National University \\ Bundang Hospital, Seongnam; \\ 'Department of Obstetrics and \\ Gynecology, Kangwon National \\ University Hospital, School of \\ Medicine, Kangwon National \\ University, Chuncheon, Korea
}

Received: 10 February 2019

Revised: 23 April 2019

Accepted: 14 May 2019

Correspondence to

Jee Yoon Park, MD

Department of Obstetrics and

Gynecology, Seoul National

University Bundang Hospital, 82

Gumi-ro 173beon-gil, Bundang-gu,

Seongnam 13620, Korea

Tel: +82-31-787-7266

Fax: +82-31-787-4054

E-mail:89492@snubh.org

Copyright@ 2019 by The Korean Society of Perinatology

This is an Open Access article distributed under the terms of the Creative Commons Attribution Non-Commercial License (http://creativecommons.org/ license/by-nc/4.0/), which permits unrestricted non-commercial use, distribution, and reproduction in any medium, provided that the original work is properly cited.
Objective: The aim of this study was to determine the association of respiratory morbidity and assisted ventilation rates in late preterm births after premature rupture of membranes (PROM).

Methods: This retrospective cohort study included 584 consecutive women admitting due to PROM and delivered between $34+0$ and $36+6$ weeks of gestation at Seoul National University Bundang Hospital from August 2008 to July 2018. Multifetal pregnancies and intrauterine fetal demise were excluded. Primary outcome included the use of assisted ventilation and neonatal respiratory complications and a multivariable analysis was also performed.

Results: 1) The proportions of neonates receiving assisted ventilation were significantly decreased as the gestational age at delivery advanced ( $P$-trend $<0.05$ for all) in late preterm births after PROM. 2) The incidences of respiratory distress syndrome were $14 \%, 7 \%$, and $3 \%$ for preterm births in the 34 th, 35 th, and 36th week of gestation ( $P$-trend $=0.007$ ), respectively. 3 ) The gestational age at delivery was associated with the rates of positive pressure ventilation immediately after birth, neonatal intensive care unit (NICU) admission, minor assisted ventilators in preterm births with PROM after 34 weeks of gestation when analyzed by multivariate logistic regression. 4) The interval from PROM to delivery was independently associated with the rate of NICU admission (odds ratio, 1.04; $95 \%$ confidence interval, 1.01-1.06).

Conclusion: Although late preterm births seem to be less severe than earlier gestation, gestational age at delivery is still an important factor for neonatal respiratory complications and for the use of assisted ventilation, even when the pregnancy is complicated with PROM.

Key Words: Premature birth, Fetal membranes, Premature rupture, Respiratory distress syndrome, newborn

\section{Introduction}

Preterm birth before 37 weeks has been an obstetrical challenge for a long time. Among preterm births, late preterm babies who were born after 34 weeks have been thought to present with relatively better outcomes than those delivered earlier because sufficient maturation of the fetal lung is known to be accomplished around 34 weeks of gestation. ${ }^{1-4}$ However, physicians experience neonatal morbidities, especially from respiratory complications, more commonly than expected in clinical practice.

A current systemic meta-analysis reviewed the morbidities of late preterm births and showed that there was a higher risk for respiratory morbidities such as respiratory distress syndrome (RDS), congenital pneumonia, pneumothorax, persistent pulmonary hypertension, transient tachypnea of the newborn, and apnea when using mechanical ventilation. ${ }^{5}$ Wang 
et $\mathrm{al}^{6}{ }^{6}$ reported that the incidence of hypoglycemia, jaundice, feeding difficulties and temperature instability was increased in late preterm infants compared to term infants. Several studies reported that infants born in the late preterm period are more likely to develop long-term complications including neuropsychiatric developmental delay and poor fetal growth. ${ }^{7-11}$

Therefore, the prolongation of pregnancy should be the definite goal for patients with a risk of preterm birth in the late preterm period. For certain fetal or maternal indications, the indicated delivery is considered, and many guidelines recommend the optimal timing of delivery in several circumstances. In especially late preterm period, patients and their family members expect their babies would have less problems than early preterm babies, however in clinical circumstances, late preterm babies frequently experience respiratory difficulties. The rates of specific respiratory complications or ventilator supports are not sufficient to explain or counsel to patients.

Premature rupture of membranes (PROM) is one of the major threats of preterm birth. ${ }^{12}$ It complicates $1-5 \%$ of all pregnancies $^{13-15}$ and is highly associated with both perinatal and maternal morbidity and mortality. ${ }^{16-18}$ Traditionally intended delivery beyond 34 weeks of gestation has been considered for patients presented with PROM. ${ }^{1,19}$ It is evident from the recommendation of guidelines that being exposed to the condition of ruptured membranes has been known to be highly associated with the risk of intra-amniotic infection, chorioamnionitis, umbilical cord compression and adverse neonatal morbidities such as early onset sepsis. ${ }^{20-22}$

However, a recent review by Cochrane revealed that planned delivery in PROM patients prior to 37 weeks was not associated with improved neonatal morbidity or reduced perinatal mortality. ${ }^{23}$ In addition, van der Ham et al. ${ }^{24,25}$ reported on randomized trials showing that immediate intended delivery did not reduce the risk of neonatal sepsis (relative risk, 0.66; 95\% confidence interval [CI], 0.30-1.5), and other neonatal outcomes were not different compared to those who underwent expectant management. The above study suggested that expectant management could be advocated in late preterm PROM if signs of infection did not develop. $^{25}$

The aim of this study was to determine the incidence of neonatal respiratory morbidities and the use of assisted ventilation utilities according to the gestational age at delivery and the latency from rupture to delivery in late preterm births after PROM.

\section{Methods}

\section{Study design}

A retrospective study was performed on consecutive women who had delivered between $34+0$ weeks and $36+6$ weeks at Seoul National University Bundang Hospital from August 2008 to July 2018. Among them, the pregnancies who were administered for a diagnosis of preterm PROM were identified. Multifetal pregnancies and intrauterine fetal demise were excluded. The study protocol was approved by the Institutional Review Board (IRB) of Seoul National University Bundang Hospital (IRB No. E-1705396-103).

\section{The outcomes of the pregnancies and neonates}

Data including maternal and neonatal outcomes were collected. Maternal characteristics included age, parity, use of any tocolytics, use of progesterone for prevention of preterm birth before PROM, antenatal steroid administration, use of intravenous oxytocin, the mode of delivery, and gestational age at the diagnosis of ruptured membranes and at delivery. Placental pathologic reports were collected to evaluate the presence of histologic chorioamnionitis and funisitis.

Basic information on the newborns such as birthweight, sex, Apgar scores, the presence of meconium, and major anomalies were collected. The outcome variables were the rate of neonatal intensive care unit (NICU) admission, the use of assisted ventilation, and respiratory morbidities including RDS, bronchopulmonary dysplasia (BPD), frequent apnea, transient tachypnea, and pneumothorax. Composite respiratory morbidity was defined as the presence of treatment for at least one of above respiratory presentations or diseases.

The definition of RDS was the presence of all of the following: clinical signs of respiratory distress, supplemental oxygen requirement $\left(\mathrm{FiO}_{2}>0.2\right)$, NICU admission for further respiratory support, diagnostic radiographic findings of reticular patterns and air bronchograms. Transient tachypnea was diagnosed when tachypnea had resolved in less than 72 hours with a normal radiography or showed increased perihilar interstitial markings in the infantogram. 
The use of positive pressure ventilation through a facial mask and the application of an intubation procedure immediately after delivery were identified. The assisted ventilation utilities were divided into three modalities: nasal continuous positive airway pressure (CPAP), high-flow therapy, and mechanical ventilator through intubation. The management on ventilatory support was decided by neonatologists.

\section{Statistical analysis}

Continuous variables were compared using the Mann-Whitney Utest; proportions were compared using the Fisher's exact test. The Jonckheere-Terpstra test for continuous variables and a linear-by-linear association for categorical variables were used when the data were analyzed according to trend as the gestational age at delivery changed. Multivariate logistic regression was used to determine factors associated with neonatal respiratory complications and with the use of assisted ventilation utilities adjusting for confounding variables that could affect the outcomes. A $P$-value $<0.05$ was considered statistically significant. The analysis was done with SPSS, version 22 (IBM SPSS Inc., Armonk, NY, USA).

\section{Results}

A total of 584 women met the inclusion criteria. Table 1 shows the baseline characteristics of the patients according to the ges- tational age at delivery. As the gestational age of delivery was earlier, the use of tocolytics to prolong pregnancy was higher. Tocolytics are not recommended to be used after 34 weeks of gestation. There were statistically significant trends in the differences of the rate of antenatal steroid administration and the interval from the rupture of membranes to the delivery ( $P$-trend $<0.05$ ). Because the study population included patients from 2008 and steroid administration had not been routinely performed before the change of recommendation which currently suggests the administration of steroids to stimulate fetal lung maturation even to late preterm births, the proportions of steroid administration were not as high as the current data. The pathologic findings such as acute histologic chorioamnionitis and funisitis tended to be found in decreased incidence as the gestational age of delivery advanced (both $P$-trend $<0.05$ ).

Table 2 reveals the neonatal outcomes according to the gestational age at delivery. As the gestational age at delivery advanced, the rates of low Apgar scores in 1 and 5 minutes decreased significantly. Assisted ventilation with positive pressure ventilation was performed in $23.6 \%$ (52/220) of the neonates born between $34+0$ and $34+6$ weeks while the rates remarkably decreased in neonates born after the 35 and 36 weeks (7.8\% [17/217] and 3.4\% [5/145], respectively, $P$-trend $<0.001$ ). The intubation procedure was done in 8 neonates: six in the 34 -weekgroup and one in the 35- and 36-week-group each.

The rate of administration was over $90 \%$ in the 34 -weekgroup, and it decreased below $10 \%$ when the gestational age

Table 1. Clinical Characteristics and Pregnancy Outcomes of the Study Population according to Gestational Age at Delivery

\begin{tabular}{|c|c|c|c|c|}
\hline & Delivered in 34 weeks $(n=221)$ & Delivered in 35 weeks $(n=218)$ & Delivered in 36 weeks ( $n=145$ ) & $P$-trend \\
\hline Age (years) & $32(20-43)$ & $31(15-41)$ & $33(23-44)$ & 0.409 \\
\hline Nulliparity & $55.7 \%(123 / 221)$ & $64.7 \%(141 / 218)$ & $55.9 \%(81 / 145)$ & 0.755 \\
\hline Antenatal steroid administration & $27.1 \%(60 / 221)$ & $9.6 \%(21 / 218)$ & $4.1 \%(6 / 145)$ & $<0.001$ \\
\hline Use of intravenous oxytocin & $51.1 \%(113 / 221)$ & $49.5 \%(108 / 218)$ & $44.1 \%(64 / 145)$ & 0.207 \\
\hline Gestational age at delivery (weeks) & $34.4(34.0-34.9)$ & $35.4(35.0-35.9)$ & $36.4(36.0-36.9)$ & $<0.001$ \\
\hline Interval from ROM to delivery (hours) & $25.8(0-3402.5)$ & $14.5(0.1-1761.3)$ & $10.3(0.1-1469.7)$ & $<0.001$ \\
\hline Delivered in 24 hours & $48.9 \%(108 / 221)$ & $67.0 \%(146 / 218)$ & $79.3 \%(115 / 145)$ & $<0.001$ \\
\hline Delivered in 48 hours & $63.8 \%(141 / 221)$ & $82.6 \%(180 / 218)$ & $90.3 \%(131 / 145)$ & $<0.001$ \\
\hline Cesarean section & $27.6 \%(61 / 221)$ & $28.4 \%(62 / 218)$ & $33.8 \%(49 / 145)$ & 0.228 \\
\hline Acute histologic chorioamnionitis & $22.2 \%(45 / 203)$ & $16.3 \%(33 / 203)$ & $11.3 \%(15 / 133)$ & 0.009 \\
\hline Funisitis & $7.9 \%(16 / 203)$ & $3.0 \%(6 / 203)$ & $2.3 \%(3 / 133)$ & 0.010 \\
\hline
\end{tabular}

Values are expressed as median (range) for continuous variables and percentage for categorical variables. $P$-trend was analyzed by Jonckheere-Terpstra test for continuous variables and a linear-by-linear association for categorical variables.

Abbreviation: ROM, rupture of membranes. 
at delivery reached over 36 weeks $(P$-trend $<0.001)$. Assisted ventilation including nasal CPAP, high-flow therapy, and mechanical ventilator after admission to NICU was all significantly decreased as the gestational age at delivery advanced ( $P$-trend $<0.05$ for all). The incidences of RDS were 13.6\% (30/220), 6.5\% (14/217), and 3.4\% (5/145) for 34-, 35-, and 36-week-group, respectively ( $P$-trend $<0.001$ ). The rates of apnea decreased to $3.2 \%$ (7/220), 0.5\% (1/217), and none for the 34-, 35-, and 36week-group, respectively $(P$-trend $=0.007)$. There were no significant trends or differences in BPD, transient tachypnea, and pneumothorax among the groups.

Since the recommendation of various guidelines suggest delivery when PROM is diagnosed after 34 weeks of gestation, a subgroup analysis was done only in patients who had been administered after 34 weeks. To determine the association between the interval from the rupture of membranes to the delivery and the clinical outcomes, a comparison was done between the patients who had delivered on the day of PROM and those who had delivered within 48 hours for discrete reasons such as completion of steroid administration or individual planning of $\mathrm{ex}^{-}$ pectancy decided by physicians (Table 3 ). The median values of gestational age at PROM were all 35 weeks; however, the difference between the two groups were statistically significant $(P=0.002)$. The use of intravenous oxytocin increased $(37.4 \%$ [138/369] vs. 77.5\% [62/80], $P<0.001)$, and the rate of cesarean section decreased (35.2\% [130/369] vs. 10.0\% [8/80], $P<0.001)$ in the group who had delivered within 24 hours compared to the group who delivered within 48 hours from PROM.

In the neonatal outcomes, the group who delivered later had higher rates of a low Apgar score in 1 minute (25.0\% [20/80] vs. $15.4 \%$ [57/369], $P=0.049)$ and NICU admission (53.8\% [43/80] vs. $37.2 \%$ [137/368], $P=0.008$ ) than the group who delivered in

Table 2. Neonatal Outcomes of the Study Population according to Gestational Age at Delivery

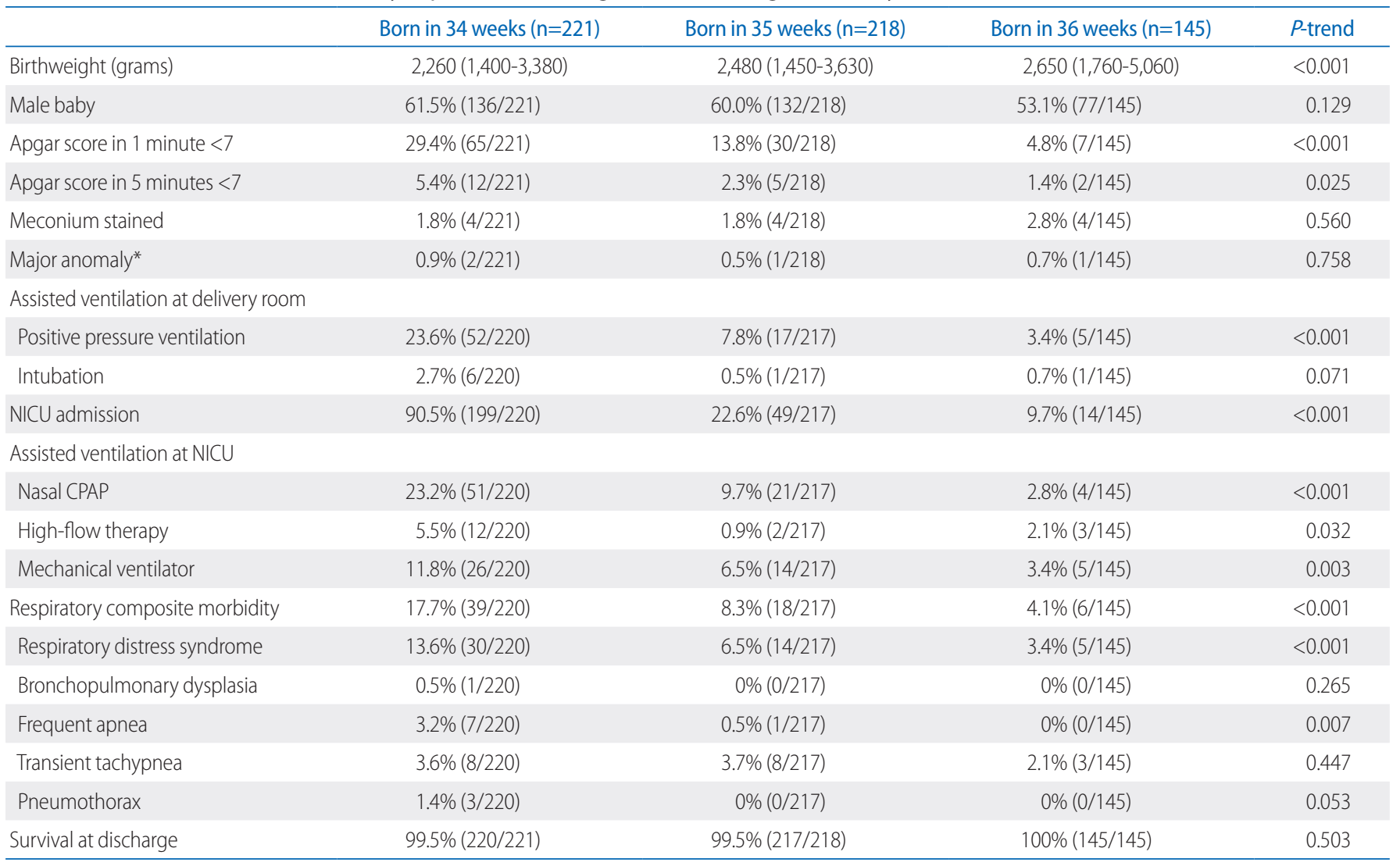

Values are expressed as median (interquartile range) for continuous variables and percentage for categorical variables. $P$-trend was analyzed by Jonckheere-Terpstra test for continuous variables and a linear-by-linear association for categorical variables. Abbreviations: NICU, neonatal intensive care unit; CPAP, continuous positive airway pressure.

*Major anomalies include three gastrointestinal anomalies (duodenal atresia, small bowel obstruction, and gastroschisis) and one heart anomaly (coarctation of aorta) 
1 day. Other variables including assisted ventilation and respiratory morbidities were comparable between the two groups.

Multivariate logistic regression analysis was done to evaluate the association of risk factors for each adverse neonatal outcome (Table 4). Advanced gestational age at delivery was independently associated with the decreased odds ratio (OR) for the rates of positive pressure ventilation immediately after birth, NICU admission, minor assisted ventilators such as nasal CPAP and/or high-flow therapy, and composite respiratory morbidity.
As the interval from the rupture of membranes to the delivery increased, admission to the NICU was slightly increased (OR, 1.04; 95\% CI, 0.1.01-1.06). The antenatal steroid administration was associated with decreased rates of mechanical ventilator use (OR, 0.32; 95\% CI, 0.12-0.91) and with the rates of composite respiratory morbidity (OR, 0.39; 95\% CI, 0.17-0.91).

Table 3. Obstetric and Neonatal Outcomes Compared according to the Interval from the ROM to the Delivery in Patients Who were Admitted for Premature ROM after 34 Weeks of Gestation

\begin{tabular}{|c|c|c|c|}
\hline & Delivered in 1 day from ROM $(n=369)$ & Delivered in 2 days from ROM $(n=80)$ & $P$-value \\
\hline Age (years) & $30(32-35)$ & $32(29-35)$ & 0.447 \\
\hline Nulliparity & $57.2 \%(211 / 369)$ & $75.0 \%(60 / 80)$ & 0.004 \\
\hline Gestational age at ROM (weeks) & $35.4(34.9-36.1)$ & $35.2(34.5-35.8)$ & 0.002 \\
\hline Antenatal steroid administration & $7.6 \%(28 / 369)$ & $11.3 \%(9 / 80)$ & 0.268 \\
\hline Use of intravenous oxytocin & $37.4 \%(138 / 369)$ & $77.5 \%(62 / 80)$ & $<0.001$ \\
\hline Gestational age at delivery (weeks) & $35.4(34.9-36.1)$ & $35.3(34.6-35.9)$ & 0.036 \\
\hline Cesarean section & $35.2 \%(130 / 369)$ & $10.0 \%(8 / 80)$ & $<0.001$ \\
\hline Acute histologic chorioamnionitis & $12.2 \%(42 / 344)$ & $22.9 \%(16 / 70)$ & 0.024 \\
\hline Funisitis & $2.9 \%(10 / 344)$ & $2.9 \%(2 / 70)$ & $>0.999$ \\
\hline Birthweight (grams) & $2,480(2,228-2,700)$ & $2,450(2,201-2,675)$ & 0.365 \\
\hline Male baby & $57.7 \%(213 / 369)$ & $61.3 \%(49 / 80)$ & 0.618 \\
\hline Apgar score in 1 minute $<7$ & $15.4 \%(57 / 369)$ & $25.0 \%(20 / 80)$ & 0.049 \\
\hline Apgar score in 5 minutes $<7$ & $3.3 \%(12 / 369)$ & $3.8 \%(3 / 80)$ & 0.737 \\
\hline Meconium stained & $1.6 \%(6 / 369)$ & $1.3 \%(1 / 80)$ & $>0.999$ \\
\hline Major anomaly* & $0.8 \%(3 / 369)$ & $1.3 \%(1 / 80)$ & 0.545 \\
\hline \multicolumn{4}{|l|}{ Assisted ventilation at delivery room } \\
\hline Positive pressure ventilation & $8.7 \%(32 / 368)$ & $10.0 \%(8 / 80)$ & 0.669 \\
\hline Intubation & $0.8 \%(3 / 368)$ & $0 \%(0 / 80)$ & $>0.999$ \\
\hline NICU admission & $37.2 \%(137 / 368)$ & $53.8 \%(43 / 80)$ & 0.008 \\
\hline \multicolumn{4}{|l|}{ Assisted ventilation at NICU } \\
\hline Nasal CPAP & $12.5 \%(48 / 368)$ & $13.8 \%(11 / 80)$ & 0.715 \\
\hline High-flow therapy & $1.9 \%(7 / 368)$ & $5.0 \%(4 / 80)$ & 0.114 \\
\hline Mechanical ventilator & $6.3 \%(23 / 368)$ & $10.0 \%(8 / 80)$ & 0.228 \\
\hline Respiratory composite morbidity & $10.9 \%(40 / 368)$ & $16.3 \%(13 / 80)$ & 0.183 \\
\hline Respiratory distress syndrome & $8.4 \%(31 / 368)$ & $12.5 \%(10 / 80)$ & 0.283 \\
\hline Bronchopulmonary dysplasia & $0.3 \%(1 / 368)$ & $0 \%(0 / 80)$ & $>0.999$ \\
\hline Frequent apnea & $0.8 \%(3 / 368)$ & $3.8 \%(3 / 80)$ & 0.073 \\
\hline Transient tachypnea & $3.0 \%(11 / 368)$ & $3.8 \%(3 / 80)$ & 0.723 \\
\hline Pneumothorax & $0 \%(0 / 368)$ & $0 \%(0 / 80)$ & NA \\
\hline
\end{tabular}

Values are expressed as median (range) for continuous variables and percentage for categorical variables.

Abbreviations: ROM, rupture of membranes; NICU, neonatal intensive care unit; CPAP, continuous positive airway pressure; NA, not available.

*Major anomalies include three gastrointestinal anomalies (duodenal atresia, small bowel obstruction, and gastroschisis) and one heart anomaly (coarctation of aorta). 
Table 4. The Association between the Latency from the ROM to the Delivery and the Neonatal Respiratory Complications or Ventilator Use in Patients Who were Admitted for Premature ROM after 34 Weeks of Gestation and Delivered in Two Days

\begin{tabular}{|c|c|c|}
\hline & OR $(95 \% \mathrm{Cl})$ & $P$-value \\
\hline \multicolumn{3}{|l|}{ PPV immediately after delivery } \\
\hline Interval from ROM to delivery (hours) & $1.01(0.98-1.04)$ & 0.660 \\
\hline Gestational age at delivery & $0.49(0.30-0.80)$ & 0.004 \\
\hline Antenatal corticosteroids & $0.70(0.24-2.02)$ & 0.509 \\
\hline Acute histologic chorioamnionitis & $0.95(0.37-2.47)$ & 0.918 \\
\hline Cesarean section & $1.62(0.75-3.49)$ & 0.217 \\
\hline \multicolumn{3}{|l|}{ NICU admission } \\
\hline Interval from ROM to delivery (hours) & $1.04(1.01-1.06)$ & 0.004 \\
\hline Gestational age at delivery & $0.11(0.07-0.17)$ & $<0.001$ \\
\hline Antenatal corticosteroids & $0.39(0.15-1.00)$ & 0.051 \\
\hline Acute histologic chorioamnionitis & $1.00(0.49-2.03)$ & 0.994 \\
\hline Cesarean section & $2.04(1.15-3.62)$ & 0.015 \\
\hline \multicolumn{3}{|l|}{ Nasal CPAP and/or high-flow therapy } \\
\hline Interval from ROM to delivery (hours) & $1.02(1.00-1.05)$ & 0.104 \\
\hline Gestational age at delivery & $0.38(0.25-0.58)$ & $<0.001$ \\
\hline Antenatal corticosteroids & $0.47(0.20-1.11)$ & 0.085 \\
\hline Acute histologic chorioamnionitis & $1.73(0.70-4.24)$ & 0.234 \\
\hline Cesarean section & $1.65(0.86-3.19)$ & 0.132 \\
\hline \multicolumn{3}{|l|}{ Use of mechanical ventilator } \\
\hline Interval from ROM to delivery (hours) & $1.03(1.00-1.06)$ & 0.091 \\
\hline Gestational age at delivery & $0.66(0.40-1.09)$ & 0.106 \\
\hline Antenatal corticosteroids & $0.32(0.12-0.91)$ & 0.032 \\
\hline Acute histologic chorioamnionitis & $7.40(0.95-57.76)$ & 0.056 \\
\hline Cesarean section & $1.59(0.69-3.69)$ & 0.279 \\
\hline \multicolumn{3}{|l|}{ Composite respiratory morbidity } \\
\hline Interval from ROM to delivery (hours) & $1.02(1.00-1.05)$ & 0.081 \\
\hline Gestational age at delivery & $0.45(0.29-0.69)$ & $<0.001$ \\
\hline Antenatal corticosteroids & $0.39(0.17-0.91)$ & 0.030 \\
\hline Acute histologic chorioamnionitis & $2.44(0.88-6.80)$ & 0.088 \\
\hline Cesarean section & $1.32(0.67-2.62)$ & 0.428 \\
\hline
\end{tabular}

Abbreviations: ROM, rupture of membranes; $\mathrm{OR}$, odds ratio; $\mathrm{Cl}$, confidence interval; PPV, positive pressure ventilation; NICU, neonatal intensive care unit; CPAP, continuous positive airway pressure.

\section{Discussion}

\section{Principle findings of the study}

1) The proportions of neonates who had received assisted ventilation including nasal CPAP, high-flow therapy, and mechanical ventilator were all significantly decreased as the gestational age at delivery advanced ( $P$-trend $<0.05$ for all) in late preterm births after PROM. 2) The incidences of RDS were $14 \%, 7 \%$, and $3 \%$ for preterm births in the 34th, 35th, and 36th week of gestation $(P$-trend $=0.007) .3$ ) The gestational age at delivery was associated with the rates of positive pressure ventilation immediately after birth, NICU admission, minor assisted ventilators such as nasal CPAP and/or high-flow therapy, and composite respiratory morbidity in preterm births with PROM after 34 weeks of gestation when analyzed by multivariate logistic regression. 4) The interval from PROM to delivery was independently associated with the rate of NICU admission (OR, 1.04; 95\% CI, 1.01-1.06).

\section{Concerns about optimal timing of delivery in the late preterm period after PROM}

A survey once reported about $70 \%$ of physicians prefer to proceed to delivery when a patient presents with PROM after 34 weeks of gestation. ${ }^{26}$ Late preterm neonates have been considered sufficiently mature to be treated similarly to term infants. While many of these infants have shown positive outcomes, evidence suggesting significant maturation occurs during the last weeks of gestation has been reported and supports the considerable risk of adverse outcomes in neonates born before 37 weeks of gestation. ${ }^{27,28}$

Most patients and their family members want to prolong pregnancy until the term period. Physicians suggest proceeding to delivery for preterm PROM patients in the late preterm period with an explanation of the complications such as chorioamnionitis and the reassurance of better morbidity than those in earlier gestation. However, if the neonates are admitted to the NICU and receive assisted ventilation, patients tend to be suspicious about the decision to delivery.

This study revealed, even in the late preterm period, gestational age at birth is a critical factor for neonatal morbidities and assisted ventilation use in preterm births complicated with PROM. A number of guidelines recommend not to prolong pregnancy after 34 weeks of gestation in patients with PROM to avoid the development of chorioamnionitis and further neonatal complications such as early-onset sepsis. However, this study demonstrated relatively high rates of assisted ventilation use and neonatal respiratory problems including major and minor ones. This result carefully suggests that expectant management rather than indicated delivery for late preterm patients with PROM 
would decrease the chance of respiratory complications if close monitoring for signs of chorioamnionitis such as fever is combined with it.

Indeed, current studies have challenged the traditional recommendations with reporting better or similar outcomes for expectant management in late preterm PROM patients when compared with indicated delivery immediately after 34 weeks of gestation. van der Ham et al., ${ }^{25}$ demonstrated that the induction of labor did not reduce the risk of neonatal sepsis after PROM. The trial advocated expectant management with the identification of a high-risk subgroup for infection in later preterm PROM because it could potentially decrease neonatal complications from prematurity and the costs for the intensive care of neonates. ${ }^{29}$

There are a few studies showing the adverse outcomes of expectant management in later preterm PROM patients. Nayot et al., ${ }^{3}$ reported no clear benefit of expectant management compared to elective induction of delivery in patients who had presented PROM after 34 weeks of gestation. Moreover, they demonstrated a significant increase in the incidence of necrotizing enterocolitis and longer admission days in the expectant management group than in the indicated delivery group.

\section{The detailed information for counseling patients who pre- sent with PROM in late preterm and have a pending delivery}

The rates of receiving positive pressure ventilator immediately after delivery were $24 \%, 8 \%$, and $3 \%$ in neonates born in the $34-$, $35-$, and 36-week, respectively. The proportion of intubation at the delivery room was about $3 \%$ in preterm births in the 34 th week and decreased to under $1 \%$ in the 35th and 36th week of gestation. The rates of relatively non-invasive assisted ventilation such as nasal CPAP and/or high-flow therapy were 23\% and $6 \%$ for neonates born in the 34th week. Both the rates decreased below $10 \%$ and $1 \%$ when the gestational age at delivery advanced to 35 and 36 weeks of gestation. The mechanical ventilator use was $12 \%, 7 \%$, and $3 \%$ for the 34 th, 35th, and 36th week, respectively.

The rates of respiratory morbidity decreased when born in the 34 th, 35th, and 36th week (18\%, 8\%, and 4\%, respectively). The incidences of RDS, one of the most serious neonatal respiratory complications, decreased by about half (from $14 \%$ to $7 \%$ ) when compared between the 34th and 35th week of gestation. Apnea is a rather minor respiratory complication; nevertheless, close monitoring is likely needed when presented. The known incidence of apnea is $4-7 \%$ for late preterm and less than $1-2 \%$ in term neonates. ${ }^{30-32}$ Our study showed that the rates of frequent apnea were $3 \%, 1 \%$, and $0 \%$ for neonates born in the 34 th, 35 th, and 36th week, respectively.

\section{Strengths and limitations}

The inevitable limitation of this study is its retrospective nature; however, the study population is relatively well-purified and restricted to a single tertiary center. All the studies done to identify neonatal respiratory outcomes in late preterm births with PROM were designed with the inclusion criteria focusing on maternal status. Therefore, adjusting for various factors was necessary. This study was done with only babies who were born in the late preterm period, meaning the outcomes were analyzed in late preterm neonates themselves, not in all the babies from mothers who presented with PROM. The proportions of each assisted ventilator care or respiratory morbidities including minor problems such as apnea will be a beneficial information for clinicians who need to counsel with patients since those data are lack in late preterm babies.

Pasquier et al., ${ }^{33}$ reported similar results with our research on late preterm PROM patients. Those authors concluded that adverse neonatal outcomes were inversely related to gestational age at the time of PROM; however, the inclusion criteria for the study population were PROM cases from 24 weeks of gestation. ${ }^{33}$ Another study showed a higher rate of chorioamnionitis and neonatal sepsis in patients who had received expectant management compared to those with indicated delivery with prolongation of the latency period. ${ }^{34}$ The gestational age of PROM in this study was from 32 to 34 weeks of gestation.

Although late preterm births seem to be less severe than that of earlier gestation, gestational age at delivery is still an important factor for neonatal respiratory complications and for the use of assisted ventilation, even when the pregnancy is complicated with PROM.

\section{References}

1) Sentilhes L, Sénat MV, Ancel PY, Azria E, Benoist G, Blanc J, et al. Prevention of spontaneous preterm birth: guidelines for clinical practice from the French College of Gynaecologists and Obstetricians (CNGOF). Eur J 
Obstet Gynecol Reprod Biol 2017;210:217-24.

2) Chan $E$, Quigley MA. School performance at age 7 years in late preterm and early term birth: a cohort study. Arch Dis Child Fetal Neonatal Ed 2014;99:F451-7.

3) Nayot D, Penava D, Da Silva O, Richardson BS, de Vrijer B. Neonatal outcomes are associated with latency after preterm premature rupture of membranes. J Perinatol 2012;32:970-7.

4) Moster D, Lie RT, Markestad T. Long-term medical and social consequences of preterm birth. N Engl J Med 2008;359:262-73.

5) Teune MJ, Bakhuizen S, Gyamfi Bannerman C, Opmeer BC, van Kaam $A H$, van Wassenaer $A G$, et al. A systematic review of severe morbidity in infants born late preterm. Am J Obstet Gynecol 2011;205:374.e1-9.

6) Wang ML, Dorer DJ, Fleming MP, Catlin EA. Clinical outcomes of nearterm infants. Pediatrics 2004;114:372-6.

7) Rogers $C E$, Lenze $S N$, Luby JL. Late preterm birth, maternal depression, and risk of preschool psychiatric disorders. J Am Acad Child Adolesc Psychiatry 2013;52:309-18.

8) Woythaler MA, McCormick MC, Smith VC. Late preterm infants have worse 24-month neurodevelopmental outcomes than term infants. Pediatrics 2011;127:e622-9.

9) Mathews TJ, MacDorman MF. Infant mortality statistics from the 2006 period linked birth/infant death data set. Natl Vital Stat Rep 2010;58:131.

10) Morse SB, Zheng $H$, Tang $Y$, Roth J. Early school-age outcomes of late preterm infants. Pediatrics 2009;123:e622-9.

11) Santos IS, Matijasevich A, Domingues MR, Barros AJ, Victora CG, Barros FC. Late preterm birth is a risk factor for growth faltering in early childhood: a cohort study. BMC Pediatr 2009;9:71.

12) Goldenberg RL, Rouse DJ. Prevention of premature birth. N Engl J Med 1998;339:313-20

13) Mercer BM, Goldenberg RL, Meis PJ, Moawad AH, Shellhaas C, Das A, et al. The preterm prediction study: prediction of preterm premature rupture of membranes through clinical findings and ancillary testing. The National Institute of Child Health and Human Development Ma ternal-Fetal Medicine Units Network. Am J Obstet Gynecol 2000;183: 738-45.

14) Cox SM, Williams ML, Leveno KJ. The natural history of preterm ruptured membranes: what to expect of expectant management. Obstet Gynecol 1988;71:558-62.

15) Gibbs RS, Blanco JD. Premature rupture of the membranes. Obstet Gynecol 1982;60:671-9.

16) Mercer BM. Preterm premature rupture of the membranes. Obstet Gynecol 2003;101:178-93.

17) Furman B, Shoham-Vardi I, Bashiri A, Erez O, Mazor M. Clinical significance and outcome of preterm prelabor rupture of membranes: populationbased study. Eur J Obstet Gynecol Reprod Biol 2000;92:209-16.

18) Goldenberg RL, Nelson KG, Davis RO, Koski J. Delay in delivery: influence of gestational age and the duration of delay on perinatal outcome. Obstet Gynecol 1984;64:480-4.

19) Kuba K, Bernstein PS. ACOG practice bulletin no. 188: prelabor rupture of membranes. Obstet Gynecol 2018;131:1163-4.
20) Packard RE, Mackeen AD. Labor induction in the patient with preterm premature rupture of membranes. Semin Perinatol 2015;39:495-500.

21) Mackeen AD, Seibel-Seamon J, Muhammad J, Baxter JK, Berghella $V$. Tocolytics for preterm premature rupture of membranes. Cochrane Database Syst Rev 2014;(2):CD007062.

22) Naef RW 3rd, Allbert JR, Ross EL, Weber BM, Martin RW, Morrison JC. Premature rupture of membranes at 34 to 37 weeks' gestation: aggressive versus conservative management. Am J Obstet Gynecol 1998;178 (1 Pt 1):126-30.

23) Buchanan SL, Crowther CA, Levett KM, Middleton P, Morris J. Planned early birth versus expectant management for women with preterm prelabour rupture of membranes prior to 37 weeks' gestation for improving pregnancy outcome. Cochrane Database Syst Rev 2010;(3): CD004735.

24) van der Ham DP, van der Heyden $J$, Opmeer BC, Mulder AL, Moonen RM, van Beek JH, et al. Management of late-preterm premature rupture of membranes: the PPROMEXIL-2 trial. Am J Obstet Gynecol 2012;207: 276.e1-10.

25) van der Ham DP, Vijgen SM, Nijhuis JG, van Beek JJ, Opmeer BC, Mulder $A L$, et al. Induction of labor versus expectant management in women with preterm prelabor rupture of membranes between 34 and 37 weeks: a randomized controlled trial. PLoS Med 2012;9:e1001208.

26) Aliaga S, Price W, McCaffrey M, Ivester T, Boggess $K$, Tolleson-Rinehart $S$. Practice variation in late-preterm deliveries: a physician survey. J Perinatol 2013;33:347-51.

27) Pike KC, Lucas JS. Respiratory consequences of late preterm birth. Paediatr Respir Rev 2015;16:182-8.

28) Boyle EM, Poulsen G, Field DJ, Kurinczuk JJ, Wolke D, Alfirevic Z, et al. Effects of gestational age at birth on health outcomes at 3 and 5 years of age: population based cohort study. BMJ 2012;344:e896.

29) van der Ham DP, van Kuijk S, Opmeer BC, Willekes C, van Beek JJ, Mulder $\mathrm{AL}$, et al. Can neonatal sepsis be predicted in late preterm premature rupture of membranes? Development of a prediction model. Eur J Obstet Gynecol Reprod Biol 2014;176:90-5.

30) Hunt CE. Ontogeny of autonomic regulation in late preterm infants born at 34-37 weeks postmenstrual age. Semin Perinatol 2006;30:73-6.

31) Arnon S, Dolfin T, Litmanovitz I, Regev R, Bauer S, Fejgin M. Preterm la bour at 34--36 weeks of gestation: should it be arrested? Paediatr Perinat Epidemiol 2001;15:252-6.

32) Henderson-Smart DJ, Pettigrew AG, Campbell DJ. Clinical apnea and brain-stem neural function in preterm infants. N Engl J Med 1983;308: 353-7.

33) Pasquier JC, Picaud JC, Rabilloud M, Claris O, Ecochard R, Moret S, et al. Neonatal outcomes after elective delivery management of preterm premature rupture of the membranes before 34 weeks' gestation (DO MINOS study). Eur J Obstet Gynecol Reprod Biol 2009;143:18-23.

34) Tsafrir Z, Margolis G, Cohen Y, Cohen A, Laskov I, Levin I, et al. Conservative management of preterm premature rupture of membranes beyond 32 weeks' gestation: is it worthwhile? J Obstet Gynaecol 2015;35:58590. 